\title{
The relationship between vitamin B12 and vitamin $D$ levels and subjective cognitive complaints in patients with first episode major depressive disorder
}

\author{
Selen Isik Ulusoy ${ }^{1 \oplus}$, Bahriye Horasanli ${ }^{\circledR}{ }^{\oplus}$, Sevsen Kulaksizoglu ${ }^{3 \oplus}$ \\ 'Baskent University, Faculty of Medicine, Konya Research Hospital, Department of Psychiatry, Konya - Turkey \\ ${ }^{2}$ Baskent University, Faculty of Medicine, Konya Research Hospital, Department of Neurology, Konya - Turkey \\ ${ }^{3}$ Baskent University, Faculty of Medicine, Konya Research Hospital, Department of Biochemistry, Konya - Turkey
}

\begin{abstract}
Objective: Cognitive dysfunction is an important component of major depressive disorder (MDD). The aim of this study is to investigate the relationship between vitamin B12 and D and subjective cognitive complaints in patients with first-episode MDD.

Method: In this cross-sectional study, patients $(n=120)$ diagnosed with first-episode MDD filled out self-report questionnaires that evaluate subjective neurocognitive symptoms (Perceived Deficits Questionnaire-Depression [PDQ-D] and British Columbia Cognitive Complaints Inventory [BC-CCI]). Disease severity was evaluated using the Hamilton Depression Rating Scale (HDRS). Serum 25-OH vitamin D and vitamin B12 levels were measured using a chemiluminescence microparticle immunoassay technology.

Results: The PDQ-D and BC-CCI scores were significantly higher in patients with combined vitamin B12 and D deficiency than patients with normal vitamin levels. We found a negative correlation between vitamin B12 level and HDRS, BC-CCI and PDQ-D scores. In addition, there was a positive correlation between HDRS scores and PDQ-D and BC-CCI scores. Linear regression analysis revealed that HDRS score and vitamin B12 level have influence on PDQ-D and BC-CCI scores.

Conclusion: In this study, it was found that subjective cognitive impairment was significantly higher in MDD patients with both of vitamin B12 and vitamin D deficiency. Vitamin B12 levels were also associated with depressive symptom severity and subjective cognitive impairment. Depression severity and vitamin B12 level were found to be significant predictors of subjective cognitive impairment. Our findings highlight the importance of depression severity and vitamin B12 and D levels during routine clinical evaluation of MDD.
\end{abstract}

Keywords: Cognitive complaints, depression, vitamin B12, vitamin D

\section{INTRODUCTION}

In major depressive disorder (MDD); neurocognitive functions like psychomotor speed, attention, visual learning and memory, information processing and executive functioning deteriorate from the the first episode of the disease (1). These neurocognitive functions are evaluated objectively with various

How to cite this article: Isik Ulusoy S, Horasanli B, Kulaksizoglu S. The relationship between vitamin B12 and vitamin D levels and subjective cognitive complaints in patients with first episode major depressive disorder. Dusunen Adam The Journal of Psychiatry and Neurological Sciences 2021;34:64-72.

Correspondence: Selen Isik Ulusoy, Baskent University Faculty of Medicine, Konya Research Hospital, Department of Psychiatry, 42000 Konya - Turkey

E-mail: drselen82@gmail.com

Received: June 02, 2020; Revised: August 20, 2020; Accepted: December 10, 2020 
neurocognitive assessment instruments (2). In addition to objective cognitive impairment in MDD patients, subjective cognitive complaints like forgetfulness, inattention, slowness and indecision are very common. Most studies have focused on the relationship between subjective and objective cognitive functions using tests evaluating attention, memory, and executive functions in patients with MDD. Patients' self-evaluation of their cognitive performance doses does not generally correlate with objective cognitive measures (3-6). It has been demonstrated that underestimation of both attention and memory were predicted by higher depressive symptoms and better performance in executive function tests. It is worth mentioning that depressive symptoms have significantly stronger correlations with subjective rather than objective cognitive measures (7), and also it has been found that depressive symptoms negatively affect subjective cognition (4). Among elderly people with a major depression history, subjective memory complaint has been associated with lower total instant recall and delayed verbal recall scores after adjustment (8). Inconsistency between subjective and objective function not only in the MDD patients, but also in healthy individuals. While patients with depression tend to report more subjective complaints than those measured objectively, objective cognitive abilities of healthy individuals are worse than the subjective complaints (3).

Many studies have examined the relationship between folic acid, vitamin B12 and D levels and depression. Studies have shown that low folic acid and vitamin B12 levels increase the risk of depression, and also, vitamin D levels are lower in patients with depression than in the control group (9-12). It has been reported that B12 and vitamin D supplements for the treatment of depression contribute to the improvement of depressive symptoms $(13,14)$.

Plasma or serum homocysteine levels increase in vitamin B12 and folic acid deficiency, which are toxic to neural cells. It has been mentioned that increased plasma homocysteine is a risk factor for cognitive dysfunction and dementia (15). Most of the studies investigating the association of vitamin B12 and folic acid deficiency with cognitive functions have been conducted in elderly patients and the results are conflicting (16). Although a positive correlation was found between lower vitamin B12 and folic acid levels and mini-mental test scores in normal subjects over 60 years of age (17-19), there are also studies in which vitamin B12 has no significant effect on memory tests and word recall $(20,21)$. The risk of impaired cognitive function depended on different combinations of serum folate and vitamin B12 levels in older adults. (22) As a result, the reviews indicate an association between homocysteine levels and the onset of dementia, but it is impossible to conclude the specific association between vitamin B12 and the onset of dementia.(23) Moreover, there are limited studies on the effect of vitamin B12 on cognitive impairment in patients with depression. Among elderly population vitamin B12 status is associated with cognitive deficiency, particularly in those with depression (24). Additionally, the total plasma B12 fraction bound to transcobalamin is associated with mini-mental test scores, but did not delay recall score in elderly patients with depressive symptoms (25).

Previous reviews and meta-analyses have demonstrated the association between low vitamin $\mathrm{D}$ and the risk of cognitive impairment (26-28) and, it has been revealed that there is a stronger effect for general cognition, mental speed, and visuospatial abilities compared to memory $(29,30)$. The impact of vitamin D on the subjective perception of cognitive decline has been examined amongst geriatric patients, and vitamin $\mathrm{D}$ deficiency has been associated with the subjective cognitive complaints (31). Belzeaux et al. (32) found that patients with MDD having hypovitaminosis D (25 $\mathrm{OH}$ vitamin $\mathrm{D}<20 \mathrm{ng} / \mathrm{mL}$ ) demonstrated a higher duration of stroop interference index, meaning lower cognitive inhibition ability, suggesting that hypovitaminosis D may increase cognitive impairment in patients with MDD.

In previous studies examining the relationship between vitamin levels and cognitive performances in patients with depressive symptoms, generally objective neurocognitive test batteries were used. To the best of our knowledge, no study in the literature has examined yet the relationship between subjective cognitive complaints and vitamin B12 and D levels in patients with first-episode MDD. The aim of our study is to investigate the relationship between vitamin B12 and D and subjective cognitive complaints in patients with first-episode MDD.

\section{METHOD}

\section{Study Design and Participants}

Data were derived from a sample of 120 participants with first-episode MDD recruited from the psychiatry clinic of Başkent University Hospital in Konya. This study was conducted between October 2018 and April 2019 and only outpatients with a newly diagnosed 
MDD were included. Diagnoses for MDD were performed by an experienced psychiatrist and confirmed using the Structured Clinical Interview for DSM-IV Axis I Disorders (SCID-I) (33).

The following inclusion criteria were applied: a) 18-65 years of age b) at least 5 years of education c) unipolar depression is diagnosed first and without psychotic symptoms. d) no previous exposure to antidepressants and no medication at the inclusion. Exclusion criteria for the study were a lifetime history of any other psychiatric disorder and presence of another psychiatric disorder accompanying MDD diagnosed at the SCID interview, a history of alcohol/ substance abuse/dependence within the past 6 months, a history of central nervous system trauma, neurological disorder, any active and unstable medical problem, a history of vitamin B12 or D use in the past 6 months, and folic acid deficiency.

The present study was approved by the Başkent University Institutional Review Board and Ethics Committee (Project no: KA 18/173) and supported by the Baskent University Research Fund. Each participant signed an informed consent form in accordance with the Declaration of Helsinki.

\section{Measurement of Serum 25-hydroxyvitamin D,} Vitamin B12 and Folic Acid

Participants' venous blood samples were collected from the cubital vein and after plasma samples were separated within one hour, they were stored at $-80^{\circ} \mathrm{C}$ until the samples were analyzed.

For vitamin D laboratory analyzes, vitamin D total (25-OH-vitamin D) analysis was performed in our hospital's biochemical laboratory. Serum 25-OH vitamin $\mathrm{D}$, vitamin $\mathrm{B} 12$, and folic acid were measured using chemiluminescence microparticle immunoassay technology (Abbott Architect i2000).

$25-\mathrm{OH}$ vitamin $\mathrm{D}$ deficiency was defined as a serum level below $20 \mathrm{ng} / \mathrm{mL}$ (34). The $<200 \mathrm{pg} / \mathrm{mL}$ cut-off point was widely used for vitamin B12 deficiency (35). Therefore, the cut-off point for vitamin B12 in this study was accepted as $200 \mathrm{pg} / \mathrm{mL}$, and below $200 \mathrm{pg} / \mathrm{mL}$ was defined as vitamin B12 deficiency. Folic acid levels were measured to exclude patients with folic acid deficiency and folic acid deficiency was defined as a serum level below $3 \mathrm{pg} / \mathrm{mL}$ (36).

\section{Assessment of Depression and Subjective Cognitive Symptoms}

The first major depressive episode was diagnosed using SCID-I (33). The level of depressive symptoms was rated using HDRS (Hamilton Rating Scale for Depression). PDQ-D (Perceived Deficits QuestionnaireDepression) and BC-CCI (British Columbia Cognitive Complaints Inventory) were used to assess subjective cognitive symptoms.

Structured Clinical Interview for DSM-IV Axis I Disorders: SCID-I is a clinical interview scale developed and structured by the American Psychiatric Association for the Axis-I diagnoses (33). The study for applicability and reliability of SCID-I in Turkish was conducted by Çorapçıŏlu et al (37).

Hamilton Rating Scale for Depression (HDRS): HDRS is a 17-item scale rated by an interviewer (38). It was used to assess and rate the severity of depression in patients already diagnosed with depression. The total score was calculated by summing the answers to the questions; higher scores indicate more severe depression. It was validated in Turkish by Akdemir et al. (39).

Perceived Deficits Questionnaire-Depression (PDQ-D): This scale provides a comprehensive assessment of neurocognition with 20 items (40). Among the domains of PDQ-D are attention/ concentration, retrospective memory, prospective memory, and planning/organization. As a self-rating instrument, it has a 5-point scale for each item rated between 0 and 4 . A higher score indicates poorer functioning in neurocognition. The validity and reliability of the Turkish version of the scale were confirmed by Aydemir et al. (41).

British Columbia Cognitive Complaints Inventory (BC-CCI): This scale consists of 6 items and provides a general assessment of neurocognition in some domains in depression (42). Among these domains assessed by these 6 items, there are perceived problems with concentration, memory, difficulty in expressing thoughts, word-finding, slow thinking, and in solving problems. Subsequently, the subjects are examined with three items in terms of how they perceive and are affected by these neurocognitive deficits. Each item is rated on a 4 -point scale: $0=$ not at all, $1=$ some, $2=$ quite a bit, and $3=$ very much. The validity and reliability of the Turkish version of the scale were verified by Aydemir et al. (41).

\section{Statistical Analysis}

Statistical data were analyzed using SPSS version 21.0, with values expressed as mean \pm standard deviation. Data normality was analyzed by Kolmogorov-Smirnov test. The patients were divided into four groups according to their vitamin levels; patients with both 
vitamin B12 and D deficiency; patients with vitamin B12 deficiency only; patients with vitamin D deficiency only, and patients with normal vitamin levels. One-way analysis of variance (ANOVA) test and post hoc analysis with the Bonferroni multiple comparison tests were performed according to the Kolmogorov Smirnov test results. Correlations between the variables were investigated according to the Pearson correlation coefficient. Independent variables that correlated with subjective cognitive complaints were evaluated by linear regression analysis. Differences were considered significant at $\mathrm{p} \leq 0.05$.

The gender distribution and marital status of the patients were compared using the chi-square test. ANOVA was used to compare the age, vitamin B12, and D levels, and HDRS, BC-CCI, and PDQ-D test scores of the four groups. Bonferroni multiple comparison test was applied in the posthoc analysis in multiple comparisons of four groups. Dependent variables were age, and HDRS, BC-CCI, and PDQ-D test scores in the analysis of ANOVA with Bonferroni multiple comparison test. The correlations between subjective cognitive complaints and depressive symptoms and vitamin values were evaluated by Pearson correlation analysis, and BC-CCI, PDQ-D, HDRS scores, and vitamin B12 and D levels were taken as variables. Linear regression analysis was used to assess the significant predictors of subjective cognitive impairment measured by scales. Dependent variables were PDQ-D and BC-CCI test scores, and predictors were HDRS test score, vitamin B12 and D levels in linear regression analysis.

\section{RESULTS}

Data on age, vitamin B12 and D levels, and HDRS, BC-CCI, and PDQ-D test scores were evaluated with the Kolmogorov Smirnov test, and it was found that the significance value of all data was greater than 0.05 and showed a normal distribution $(\mathrm{p}=0.20 ; \mathrm{p}=0.19 ; \mathrm{p}=0.20$; $\mathrm{p}=0.20 ; \mathrm{p}=0.19 ; \mathrm{p}=0.20$, respectively).

A total of 127 outpatients completed the clinical assessment and had a serum $25-\mathrm{OH}$ vitamin $\mathrm{D}$, vitamin B12 and folic acid measure. 7 patients had a low serum folic acid concentration $(<3 \mathrm{pg} / \mathrm{mL})$, so they were excluded from the study. Finally, this study enrolled 120 adult patients.

The mean age of our population was $38.98 \pm 11.08$, and most of the patients were women $(72.5 \%, \mathrm{n}=87)$. Detailed demographic and clinical characteristics are shown in Table 1.
Table 1: Sociodemographic and clinical characteristics of patients $(n=120)$

\begin{tabular}{lcc} 
& Mean & SD \\
\hline $\begin{array}{l}\text { Gender (n, \%) } \\
\quad \text { Female }\end{array}$ & \\
$\quad$ Male & 33 & 72.5 \\
Age & 38.98 & 27.5 \\
Education (year) & 9.48 & 11.08 \\
Marital status (n, \%) & 3.80 \\
$\quad$ Married & 77 & \\
$\quad$ Single & 27 & 64.2 \\
$\quad$ Divorced and widowed & 16 & 22.5 \\
Vitamin B12 (pg/ml) & 290.45 & 13.3 \\
Vitamin D (ng/ml) & 16.36 & 8.36 \\
Folic acid (pg/ml) & 5.38 & 1.24 \\
HDRS & 22.38 & 7.35 \\
BC-CCI & 12.83 & 5.78 \\
PDQ-D & 31.62 & 12.70 \\
\hline
\end{tabular}

HDRS: Hamilton Rating Scale for Depression, BC-CCl: British Columbia Cognitive Complaints Inventory, PDQ-D: Perceived Deficits QuestionnaireDepression, SD: Standard deviation

Vitamin B12 deficiency $(<200 \mathrm{ng} / \mathrm{mL})$ was found in 43 patients (\% 35.83) and $25-\mathrm{OH}$ vitamin $\mathrm{D}$ deficiency $(<20 \mathrm{ng} / \mathrm{mL})$ was observed in 64 patients (\%53.33).

The patients were divided into four groups as 24 patients with both vitamin B12 and D deficiency; 19 patients with only vitamin B12 deficiency; 40 patients with only vitamin $\mathrm{D}$ deficiency and 37 patients with normal vitamin levels. There were no significant group differences in terms of gender ( $\mathrm{p}>0.05)$.

The PDQ-D and BC-CCI scores were significantly higher in patients with both vitamin $\mathrm{B} 12$ and $\mathrm{D}$ deficiency compared to patients with normal vitamin levels (respectively $\mathrm{p}<0.05$ and $\mathrm{p}<0.01$ ). There was no significant difference in PDQ-D and BC-CCI scores between patients with only B12 deficiency or only vitamin $\mathrm{D}$ deficiency and patients with normal vitamin levels. Additionally, patients with both vitamin B12 and $\mathrm{D}$ deficiency has significantly higher PDQ-D and BC-CCI scores than patients with vitamin B12 deficiency only $(\mathrm{p}<0.05$ and $\mathrm{p}<0.05$, respectively). The means and standard deviations of the comparisons for subjective cognition measures, vitamin concentrations and HDRS scores between groups are presented in Table 2.

When we examined the correlations between test scores and vitamin B12 and vitamin D levels in all patients, we found a negative correlation between the vitamin B12 level and HDRS, BC-CCI and PDQ-D 
Table 2: Comparison of data from groups using ANOVA with Bonferroni multiple comparison test

\begin{tabular}{|c|c|c|c|c|c|c|c|c|c|c|}
\hline & $\begin{array}{c}B 12(\downarrow) D(\downarrow) \\
(n=24)\end{array}$ & $\begin{array}{c}B 12(\downarrow) D(N) \\
(n=19)\end{array}$ & $\begin{array}{c}B 12(N) D(\downarrow) \\
(n=40)\end{array}$ & $\begin{array}{c}B 12(N) D(N) \\
(n=37)\end{array}$ & P $1 a 2$ & P 1 a3 & P $1 a 4$ & P $2 a 3$ & P $2 a 4$ & P 3a4 \\
\hline Age & $36.72 \pm 9.81$ & $42.08 \pm 12.99$ & $39.03 \pm 10.88$ & $39.55 \pm 11.13$ & 0.384 & 1.000 & 1.000 & 1.000 & 1.000 & 1.000 \\
\hline Vitamin B12 & $162.21 \pm 30.92$ & $185.00 \pm 10.79$ & $332.00 \pm 86.04$ & $383.64 \pm 118.57$ & 0.087 & $<0.001$ & $<0.001$ & $<0.001$ & $<0.001$ & 0.117 \\
\hline Vitamin D & $11.46 \pm 4.98$ & $26.50 \pm 6.07$ & $10.32 \pm 4.03$ & $23.60 \pm 2.55$ & $<0.001$ & 0.992 & $<0.001$ & $<0.001$ & 0.175 & $<0.001$ \\
\hline HDRS & $23.41 \pm 8.49$ & $21.63 \pm 7.12$ & $22.56 \pm 6.72$ & $20.81 \pm 6.55$ & 1.000 & 1.000 & 1.000 & 1.000 & 1.000 & 1.000 \\
\hline BC-CCI & $15.94 \pm 5.50$ & $11.07 \pm 3.90$ & $12.90 \pm 6.50$ & $10.46 \pm 4.98$ & 0.012 & 0.058 & 0.003 & 1.000 & 1.000 & 0.992 \\
\hline PDQ-D & $37.15 \pm 11.09$ & $27.23 \pm 12.85$ & $31.24 \pm 14.11$ & $26.81 \pm 9.65$ & 0.025 & 0.236 & 0.014 & 1.000 & 1.000 & 0.706 \\
\hline
\end{tabular}

HDRS: Hamilton Rating Scale for Depression, BC-CCI: British Columbia Cognitive Complaints Inventory, PDQ-D: Perceived Deficits Questionnaire-Depression, ANOVA: Analysis of variance. $B 12(\downarrow) D(\downarrow)$ : Patients with both vitamin $B 12$ and $D$ deficiency. $B 12(\downarrow) D(N)$ : Patients with only vitamin $B 12$ deficiency. $B(N) D(\downarrow)$ : Patients with only vitamin $D$ deficiency. $B(N) D(N)$ : Patients with normal vitamin levels. $P 1$ 12: $P$ value in comparison of patients with both vitamin $B 12$ and $D$ deficiency and patients with only vitamin $B 12$ deficiency. $P$ 1a3: $P$ value in comparison of patients with both vitamin $B 12$ and $D$ deficiency and patients with only vitamin $D$ deficiency. $P$ 1a4: $P$ value in comparison of patients with both vitamin $B 12$ and $D$ deficiency and patients with normal vitamin levels. $P$ 2a3: $P$ value in comparing patients with only vitamin B12 deficiency and patients with only vitamin D deficiency. P 2a4: P value in comparing patients with only vitamin B12 deficiency and patients with normal vitamin levels. $P$ 3a4: $P$ value in comparing patients with only vitamin D deficiency and patients with normal vitamin levels. ANOVA with Bonferroni multiple comparison test was performed for statistical analysis. $p<0.05$

Table 3: Pearson's correlation analysis results for correlations between HDRS, vitamin B12 and D levels, and HDRS, BC-CCI, PDQ-D test scores $(n=120)$

\begin{tabular}{|c|c|c|c|c|c|c|}
\hline & \multicolumn{2}{|c|}{ HDRS } & \multicolumn{2}{|c|}{$\mathrm{BC}-\mathrm{CCl}$} & \multicolumn{2}{|c|}{ PDQ-D } \\
\hline & $\mathbf{r}$ & $\mathbf{p}$ & $\mathbf{r}$ & $\mathbf{p}$ & $\mathbf{r}$ & $\mathbf{p}$ \\
\hline Vitamin B12 & -0.211 & 0.021 & -0.300 & 0.001 & -0.270 & 0.003 \\
\hline Vitamin D & -0.007 & 0.941 & 0.105 & 0.255 & -0.126 & 0.171 \\
\hline HDRS & 1 & - & 0.294 & 0.001 & 0.469 & $<0.001$ \\
\hline
\end{tabular}

HDRS: Hamilton Rating Scale for Depression, BC-CCl: British Columbia Cognitive Complaints Inventory, PDQ-D: Perceived Deficits Questionnaire-Depression. Pearson's correlation analysis was performed for statistical analysis. r: Pearson's correlation coefficient; $p<0.05$

Table 4: Linear regression analysis with PDQ-D and BC-CCI scores as dependent variables in patients $(n=120)$

\begin{tabular}{lcccc} 
Dependent variable & Predictors & Standardized coefficients $\boldsymbol{\beta}$ & $\mathbf{t}$ & $\mathbf{p}$ \\
\hline PDQ-D & Constant & & 5.599 & -2.228 \\
& Vitamin B12 & -0.177 & -1.195 & 0.028 \\
& Vitamin D & -0.094 & 5.530 & 0.235 \\
BC-CCI & HDRS & 0.438 & 2.594 & $<0.001$ \\
& Constant & & -1.870 & 0.000 \\
& Vitamin B12 & -0.112 & 0.305 & 0.004 \\
& Vitamin D & 0.018 & -1.442 & 0.509 \\
\hline
\end{tabular}

PDQ-D: Perceived Deficits Questionnaire-Depression, BC-CCl: British Columbia Cognitive Complaints Inventory, HDRS: Hamilton Rating Scale for Depression. Linear regression analysis was performed for statistical analysis. $p<0.05$

scores $(\mathrm{r}=-0.21, \mathrm{p}<0.05 ; \mathrm{r}=-0.30, \mathrm{p}<0.01 ; \mathrm{r}=-0.27 \mathrm{p}$ : $\mathrm{p}<0.01$, respectively) Also, there was a positive correlation between HDRS scores and PDQ-D and BC-CCI scores $(r=0.46, p<0.001 ; \mathrm{r}=0.29, \mathrm{p}<0.01$, respectively). The correlations between test scores and vitamin levels are presented in Table 3.

In the linear regression analysis performed to verify the relationships found in the correlation analysis, it was found that HDRS score and vitamin B12 level had an effect on the PDQ-D and BC-CCI scores (Table 4).

\section{DISCUSSION}

The present study revealed that first-episode MDD patients with both vitamin B12 and vitamin D deficiency had subjective cognitive impairment compared to MDD patients with normal vitamin levels. In addition, we found an association between subjective cognitive impairment with vitamin B12 level and depression severity in all patients by correlation analysis. In linear regression analysis, depression 
severity and vitamin B12 levels were significant predictors of subjective cognitive impairment.

We divided the patients into subgroups according to their vitamin levels. When the mean test scores measuring subjective cognitive complaints were compared, the scores of the patients with vitamin B12 deficiency only or vitamin D deficiency were higher than the patient group with normal vitamin levels, but no statistically significant difference was found. A statistically significant difference was found in these test scoresbetween the patient group with both vitamin B12 and $\mathrm{D}$ deficiency and the patient group with normal vitamin levels. There was no significant difference in subjective cognitive complaints compared to patients with only vitamin $\mathrm{B} 12$ or only vitamin $\mathrm{D}$ deficiency compared to patients with normal vitamin levels. These findings suggest that the coexistence of low levels of B12 and D vitamins may affect subjective cognitive symptoms in MDD patients, but further studies are needed an our results need to be clarified.

In studies examining the effect of folic acid and vitamin B12 levels on depression in the literature, it was concluded that folic acid and vitamin B12 deficiencies are associated with an increased risk of depression (9, 10). Findings in a recent systematic review have also shown that the short-term use of folic acid and Vitamin B12 (days to a few weeks) does not contribute to the improvement of depressive symptoms in adults with MDD, but more longer-term consumption (from several weeks to years) may reduce the risk of relapse and the cause the onset of depressive symptoms in people at risk (14). In this study, we divided patients into four groups according to their vitamin levels, but we couldn't find any difference between the groups in terms of depressive symptom severity. This finding may be contradictory the literature, but when we examined the correlations between vitamin levels and depressive symptom severity in all patients; there was a negative correlation between vitamin B12 level and the severity of depressive symptoms. This correlation is consistent with the studies investigating the relationship between vitamin B12 level and depressive symptoms in the literature.

In observational studies on the relationship between depression and 25-OH vitamin D levels, it was reported that the serum concentration of $25-\mathrm{OH}$ vitamin $\mathrm{D}$ was lower in people with depression compared to controls $(11,43)$. A systematic review found that $25-\mathrm{OH}$ vitamin $\mathrm{D}$ supplementation had a significant effect in reducing depressive symptoms for participants with clinically significant depressive symptoms (13). In this study, we couldn't found an association between 25-OH vitamin $\mathrm{D}$ level and depressive symptom severity. Since our sample consists only of patients diagnosed with MDD and cannot be compared with the healthy control group, a similar relationship to literature may not be found.

There is growing evidence that cognitive dysfunction in affective disorder impacts negatively on patients' quality of life $(44,45)$. Most of the patients with MDD present with cognitive complaints, therefore cognitive dysfunction seems to represent an important treatment goal. In recent studies, there was a discrepancy between objective and subjective cognition in adults with MDD $(4,7)$. Serra-Blasco et al. (7) subdivided MDD patients into three groups with remission, partial remission and acute episode, and found that acute MDD subgroup had lower objective and subjective cognitive scores in attention, memory and executive functions than subgroups with remission and partial remission. Depressive symptoms correlated negatively with the objective but especially with the subjective cognition (7). In studies investigating the relationship between the severity of depression and subjective cognitive complaints revealed that depression severity could predict subjectively the experienced cognitive function $(4,6)$. In this study, there is a positive correlation between depressive symptom severity and subjective cognitive complaints, and also depression severity was one of the predictors of subjective cognitive impairment. These are consistent with the evidence that cognitive problems subjectively experienced in patients with unipolar depression may represent depressive symptom severity rather than objective cognitive deficits.

The effect of vitamin B12 on cognitive functions has generally been investigated in elderly patients and the results of these studies are conflicting. While in a review Moore et al. revealed that low vitamin B12 levels are associated with neurodegenerative disease and cognitive impairment (46), there are also reviews that the current evidence is insufficient to draw clear conclusions regarding the association between vitamin B12 and cognitive decline or dementia. $(16,47)$ Consequently, the results of a meta-analysis conducted in 2013 suggest an association between homocysteine (a by-product of $B$ vitamins) levels and the onset of dementia, but not with vitamin B12 (23). In a limited number of studies in elderly patients with depressive symptoms show that vitamin B 12 deficiency increases cognitive impairment $(24,25)$. We couldn't find a study In the literature investigating the effect of vitamin B 12 on subjective cognitive symptoms in adult patients with MDD. In 
this study, it was observed that there was a positive correlation between vitamin B12 level and the severity of subjective cognitive complaints, and vitamin B12 level have also an effect on subjective cognitive complaints in regression analysis.

Vitamin D deficiency has been associated with worse cognitive performance and cognitive decline in many studies, including studies of patients with MDD $(26,32)$. Studies examining the relationship between subjective cognitive complaints and vitamin D status in elderly patients concluded that memory problems in general and daily life are associated with vitamin $\mathrm{D}$ insufficiency and that higher vitamin D levels are associated with decreased problems memorizing new information $(31,48)$. In this study, no correlation was found between the severity of subjective cognitive complaints and vitamin D level. When a comparison was made between groups, subjective cognitive complaints test scores were found to be significantly higher in patients with both of vitamin B12 and D deficiency than patients with vitamin B12 deficiency alone. In addition, it was found that patients with vitamin $\mathrm{D}$ deficiency having normal vitamin $\mathrm{B}$ levels had higher test scores for subjective cognitive complaints than patient with normal vitamin levels, but there was no statistically significant difference between the groups. In the light of these results of this study, it is difficult to say that vitamin D deficiency has no effect on subjective cognitive complaints. Further studies focusing on investigating the relationship between vitamin $\mathrm{D}$ deficiency and both objective and subjective cognitive impairments in patients with MDD are needed.

Our results need to be interpreted in the light of various limitations. First of all, we included medicationfree patients with a first major depressive episode, and we did not use an anxiety scale to exclude anxiety with comorbid depression. Additional studies with patients with recurrent depression, chronic depression, and anxiety with comorbid depression may be useful to elucidate the relationship between disease and vitamin levels. Also; since the Turkish validity and reliability study for the structured clinical interview for DSM-5 axis I disorders was not published when we started this study, we used a structured clinical interview for DSMIV axis I disorders for the psychiatric evaluation. Moreover, cross-sectional design of this study does not allow us to draw a causal conclusion regarding the relationship between subjective cognitive complaints and vitamin levels. As another limitation, evaluation of subjective cognitive symptoms together with objective cognitive impairment compared to a healthy control group may be valuable to determine the effect of vitamin $\mathrm{B} 12$ and vitamin $\mathrm{D}$ levels on cognitive symptoms. However, as previous studies have been conducted between objective cognitive impairment and vitamin levels, we have given priority to subjective cognitive complaints, which are part of daily routine clinical practice. Finally, it is also important to note that the study participants were mostly women. Thus, the study results may represent more for women than men.

In conclusion, in previous studies, patients with MDD differ in subjective and objective cognitive deficits and tend to report more subjective complaints than those measured objectively (3). During the psychiatric evaluation, patients with MDD often complain of subjective complaints about cognitive functions. In the literature, both vitamin B12 and D have been associated with depression severity and objective cognitive impairment. In this study, we revealed that MDD patients with both vitamin B12 and $\mathrm{D}$ deficiency have more subjective cognitive impairment than patients with normal vitamin levels. Vitamin B12 level and depression severity were important predictors of subjective cognitive impairment. In patients with first-episode MDD, following up the Vitamin B12 and D levels, along with the depression severity, would certainly be more useful to achieve improvements in subjective cognitive complaints.

\begin{tabular}{|l|l|l|}
\hline \multicolumn{4}{|l|}{ Contribution } & Author Initials \\
\hline \multirow{3}{*}{ Category 1} & Concept/Design & S.I.U., B.H., S.K. \\
\cline { 2 - 3 } & Data acquisition & S.I.U. \\
\cline { 2 - 3 } & Data analysis/Interpretation & S.I.U. \\
\hline \multirow{3}{*}{ Category 2} & Drafting manuscript & S.I.U. \\
\cline { 2 - 3 } & Critical revision of manuscript & S.I.U., B.H., S.K. \\
\hline \multirow{2}{*}{ Category 3} & Final approval and accountability & S.I.U., B.H., S.K. \\
\hline \multirow{3}{*}{ Other } & Technical or material support & N/A \\
\cline { 2 - 3 } & Supervision & N/A \\
\hline
\end{tabular}

Ethics Committee Approval: All procedures performed in studies involving human participants were in accordance with the ethical standards of the institutional and/or national research committee and with the 1964 Helsinki declaration and its later amendments or comparable ethical standards. The present study was approved by Baskent University Institutional Review Board and ethics Committee. (Date: 06/06/2018, Number: KA18/173)

Informed Consent: Informed consent was obtained from all individual participants included in the study.

Peer-review: Externally peer-reviewed.

Conflict of Interest: No stated conflict of interest.

Financial Disclosure: No stated funding sources. 


\section{REFERENCES}

1. Lee RS, Hermens DF, Porter MA, Redoblado-Hodge MA. A meta-analysis of cognitive deficits in first-episode major depressive disorder. J Affect Disord 2012; 140:113-124.

2. Bakkour N, Samp J, Akhras K, El Hammi E, Soussi I, Zahra F, et al. Systematic review of appropriate cognitive assessment instruments used in clinical trials of schizophrenia, major depressive disorder and bipolar disorder. Psychiatry Res 2014; 216:291-302.

3. Schwert C, Stohrer M, Aschenbrenner S, Weisbrod M, Schröder A. Biased neurocognitive self-perception in depressive and in healthy persons. J Affect Disord 2018; 232:96-102.

4. Srisurapanont M, Suttajit S, Eurviriyanukul K, Varnado P. Discrepancy between objective and subjective cognition in adults with major depressive disorder. Sci Rep 2017; 7:3901.

5. Mohn C, Rund BR. Neurocognitive profile in major depressive disorders: relationship to symptom level and subjective memory complaints. BMC Psychiatry 2016; 16:108.

6. Svendsen AM, Kessing LV, Munkholm K, Vinberg M, Miskowiak $\mathrm{KW}$. Is there an association between subjective and objective measures of cognitive function in patients with affective disorders? Nord J Psychiatry 2012; 66:248-253.

7. Serra-Blasco M, Torres IJ, Vicent-Gil M, Goldberg X, NavarraVentura G, Aguilar E, et al. Discrepancy between objective and subjective cognition in major depressive disorder. Eur Neuropsychopharmacol 2019;29:46-56.

8. Chu CS, Sun IW, Begum A, Liu SI, Chang CJ, Chiu WC, et al. The association between subjective memory complaint and objective cognitive function in older people with previous major depression. PLoS One 2017; 12:e0173027.

9. Gilbody S, Lightfoot T, Sheldon T. Is low folate a risk factor for depression? A meta-analysis and exploration of heterogeneity. J Epidemiol Community Health 2007; 61:631-637.

10. Almeida OP, McCaul K, Hankey GJ, Norman P, Jamrozik K, Flicker L. Homocysteine and depression in later life. Arch Gen Psychiatry 2008; 65:1286-1294.

11. Hoogendijk WJ, Lips P, Dik MG, Deeg DJ, Beekman AT, Penninx BW. Depression is associated with decreased 25-hydroxyvitamin $\mathrm{D}$ and increased parathyroid hormone levels in older adults. Arch Gen Psychiatry 2008; 65:508-512.

12. Belzeaux R, Boyer L, Ibrahim EC, Féron F, Leboyer M, Fond G. Mood disorders are associated with a more severe hypovitaminosis D than schizophrenia. Psychiatry Res 2015; 229:613-616.

13. Shaffer JA, Edmondson D, Wasson LT, Falzon L, Homma K, Ezeokoli $\mathrm{N}$, et al. Vitamin D supplementation for depressive symptoms: a systematic review and meta-analysis of randomized controlled trials. Psychosom Med 2014; 76:190-196.

14. Almeida OP, Ford AH, Flicker L. Systematic review and metaanalysis of randomized placebo-controlled trials of folate and vitamin B12 for depression. Int Psychogeriatr 2015; 27:727-737.

15. Smith AD, Refsum H. Homocysteine, B Vitamins, and Cognitive Impairment. Annu Rev Nutr 2016; 36:211-239.
16. Ellinson M, Thomas J, Patterson A. A critical evaluation of the relationship between serum vitamin $\mathrm{B}$, folate and total homocysteine with cognitive impairment in the elderly. J Hum Nutr Diet 2004; 17:371-383.

17. Stewart R, Asonganyi B, Sherwood R. Plasma homocysteine and cognitive impairment in an older British African-Caribbean population. J Am Geriatr Soc 2002; 50:1227-1232.

18. Duthie SJ, Whalley LJ, Collins AR, Leaper S, Berger K, Deary IJ. Homocysteine, B vitamin status, and cognitive function in the elderly. Am J Clin Nutr 2002; 75:908-913.

19. Koike T, Kuzuya M, Kanda S, Okada K, Izawa S, Enoki H, et al. Raised homocysteine and low folate and vitamin B-12 concentrations predict cognitive decline in community-dwelling older Japanese adults. Clin Nutr 2008; 27:865-871.

20. Hassing L, Wahlin A, Winblad B, Backman L. Further evidence on the effects of vitamin B12 and folate levels on episodic memory functioning: a population based study of healthy very old adults. Biol Psychiatry 1999; 45:1472-1480.

21. Jelicic M, Jonker C, Deeg DJ. Effect of low levels of serum vitamin B12 and folic acid on cognitive performance in old age: a population-based study. Dev Neuropsychol 2001; 20:565-571.

22. Castillo-Lancellotti C, Margozzini P, Valdivia G, Padilla O, Uauy R, Rozowski J, et al. Serum folate, vitamin B12 and cognitive impairment in Chilean older adults. Public Health Nutr 2015; 18:2600-2608.

23. Health Quality Ontario. Vitamin B12 and cognitive function: an evidence-based analysis. Ont Health Technol Assess Ser 2013; 13:1-45.

24. Vogiatzoglou A, Smith AD, Nurk E, Drevon CA, Ueland PM, Vollset SE, et al. Cognitive function in an elderly population: interaction between vitamin B12 status, depression, and apolipoprotein E $\varepsilon 4$ : the Hordaland Homocysteine Study. Psychosom Med 2013; 75:20-29.

25. Garrod MG, Green R, Allen LH, Mungas DM, Jagust WJ, Haan $\mathrm{MN}$, et al. Fraction of total plasma vitamin B12 bound to transcobalamin correlates with cognitive function in elderly Latinos with depressive symptoms. Clin Chem 2008; 54:12101217.

26. Goodwill AM, Szoeke C. A systematic review and meta-analysis of the effect of low vitamin d on cognition. J Am Geriatr Soc 2017; 65:2161-2168.

27. Etgen T, Sander D, Bickel H, Sander K, Förstl H. Vitamin D deficiency, cognitive impairment and dementia: a systematic review and meta-analysis. Dement Geriatr Cogn Disord 2012; 33:297-305.

28. Balion C, Griffith LE, Strifler L, Henderson M, Patterson C, Heckman G, et al. Vitamin D, cognition, and dementia: a systematic review and meta-analysis. Neurology 2012; 79:1397-1405.

29. Annweiler C, Montero-Odasso M, Llewellyn DJ, RichardDevantoy S, Duque G, Beauchet O. Meta-analysis of memory and executive dysfunctions in relation to vitamin D. J Alzheimers Dis 2013 ; 37:147-171.

30. van der Schaft J, Koek HL, Dijkstra E, Verhaar HJ, van der Schouw YT, Emmelot-Vonk MH. The association between 
vitamin D and cognition: a systematic review. Ageing Res Rev 2013;12:1013-1023.

31. Tot Babberich Ede N, Gourdeau C, Pointel S, Lemarchant B, Beauchet O, Annweiler C. Biology of subjective cognitive complaint amongst geriatric patients: vitamin $\mathrm{D}$ involvement. Curr Alzheimer Res 2015; 12:173-178.

32. Belzeaux R, Annweiler C, Bertrand JA, Beauchet O, Pichet $\mathrm{S}$, Jollant $\mathrm{F}$, et al. Association between hypovitaminosis $\mathrm{D}$ and cognitive inhibition impairment during major depression episode. J Affect Disord 2018; 225:302-305.

33. First MB, Spitzer RL, Gibbon M, Williams JBW. Structured Clinical Interview for DSM-IV Axis I Disorders, Clinician Version (SCIDCV). Washington DC: American Psychiatric Press Inc.; 1997.

34. Anglin RE, Samaan Z, Walter SD, McDonald SD. Vitamin D deficiency and depression in adults: systematic review and metaanalysis. Br J Psychiatry 2013; 202:100-107.

35. Nutritional anaemias. Report of a WHO scientific group. World Health Organ Tech Rep Ser 1968; 405:5-37.

36. Devalia V, Hamilton MS, Molloy AM; British Committee for Standards in Haematology. Guidelines for the diagnosis and treatment of cobalamin and folate disorders. Br J Haematol 2014; $166: 496-513$

37. Corapcioglu A, Aydemir O, Yildiz M, Koroglu E. Structured Clinical Interview for DSM-IV Axis I Disorders (SCID-I), Clinical Version. Ankara: Hekimler Yayin Birligi; 1999: 233236.

38. Endicott J, Cohen J, Nee J, Fleiss J, Sarantakos S. Hamilton Depression Rating Scale. Extracted from regular and change versions of the schedule for affective disorders and schizophrenia. Arch Gen Psychiatry 1981; 38:98-103.

39. Akdemir A, Orsel S, Dag İ, Turkcapar H, Iscan N, Ozbay H. Validity, reliability and clinical application of Hamilton Depression
Rating Scale (HDRS). Psikiyatri Psikoloji Psikofarmakoloji (3P) Dergisi 1996; 4:251-259.

40. Fehnel SE, Forsyth BH, DiBenedetti DB, Danchenko N, François C, Brevig T. Patient-centered assessment of cognitive symptoms of depression. CNS Spectr 2016; 21:43-52.

41. Aydemir O, Cokmus FP, Akdeniz F, Dikici DS, Balikci K. Psychometric properties of the Turkish Versions of Perceived Deficit Questionnaire - Depression and British Columbia Cognitive Complaints Inventory. Anadolu Psikiyatri Dergisi 2017; 18: 224-230.

42. Iverson GL, Lam RW. Rapid screening for perceived cognitive impairment in major depressive disorder. Ann Clin Psychiatry 2013 ; 25:135-140.

43. Walbert T, Jirikowski GF, Prüfer K. Distribution of 1,25-dihydroxyvitamin D3 receptor immunoreactivity in the limbic system of the rat. Horm Metab Res 2001; 33:525-531.

44. Dion GL, Tohen M, Anthony WA, Waternaux CS. Symptoms and functioning of patients with bipolar disorder six months after hospitalization. Hosp Community Psychiatry 1988; 39:652-657.

45. Dickerson FB, Sommerville J, Origoni AE, Ringel NB, Parente F. Outpatients with schizophrenia and bipolar I disorder: Do they differ in their cognitive and social functioning? Psychiatry Res 2001; 102:21-27.

46. Moore E, Mander A, Ames D, Carne R, Sanders K, Watters D. Cognitive impairment and vitamin B12: a review. Int Psychogeriatr 2012; 24:541-556.

47. Dangour AD, Whitehouse PJ, Rafferty K, Mitchell SA, Smith L, Hawkesworth S, et al. B-vitamins and fatty acids in the prevention and treatment of Alzheimer's disease and dementia: a systematic review. J Alzheimers Dis 2010; 22:205-224.

48. Annweiler C, Doineau L, Gerigne L, Provendier A, Karras SN, Beauchet $\mathrm{O}$, et al. Vitamin D and subjective memory complaint in community-dwelling older adults. Curr Alzheimer Res 2018; 15:664-670. 